\title{
TWO FACES OF THE HYDRODYNAMICS OF REACTION HYDROMACHINERY: THE DUALISM BETWEEN IRROTATIONALITY AND VORTEX MOTION
}

Michele FANELLI - Dr. Institute for Hydraulic and Structural Research, Italy

\section{FOREWORD}

Ever since the dawn of civilization Man has utilized natural energy sources. Apart from the muscular force of men and domesticated animals or from conversion of heat into useful work, which was not mastered until much later, the first of such sources to be harnessed on a widespread scale were -perhaps a surprising circumstance- those originated by momentum exchanges of fluid flows. Suffice it to mention:

- in the case of gas flows, the wind energy captured by sailboats and by windmills;

- in the case of liquid flows, the variations of momentum of local fields induced by controlled motion of immersed tools (oar-propelled boats); the variations of moment of gravitational forces realized in water-driven 'hydrostatic' machines (water mills); the pressure into velocity exchanges of external flow (action turbines), or the variations of moment of momentum, accompanied by pressure into velocity exchanges taking place inside internal flow, in rotating devices (reaction turbines).

Obviously, the utilization of the impulse -or of the impulse moment- generated by such exchanges was in the beginning a purely empirical achievement. The understanding of the underlying physical dynamics, as well as the capability to interpret and represent such dynamics in mathematical terms, had to wait for the wonderful Newtonian synthesis which allowed a fast development of Fluid Mechanics in qualitative and quantitative terms through the parallel invention and subsequent progress of Calculus together with the symbolic modeling of continua.

Let us here recall the two pillars of ISAAC NEWTON's “Philosophiae Naturalis Principia Mathematica" which are of interest in the present context:

Second Principle: "Mutationem motus proportionalem esse vi motrici impressae, et fieri secundum lineam rectam qua vis illa imprimitur"

Third Principle: "Actioni contrariam semper et aequalem esse reactionem: sive corporum duorum actiones in se mutuo semper esse aequales et in partes contrarias dirigi”.

From these two Principles it is but a relatively short step to desume that if a solid obstacle (be it a rigid or a deformable body, a fixed or a movable object) interacts with a fluid flow through an exchange of momentum (this last obviously intended as a vectorial quantity, so that the 'mutatio motus' could take place in amount or in direction, or both), this solid obstacle will receive an equal and vectorially opposed impulse. If the solid obstacle be a movable one, a suitable arrangement of mechanical links and degrees of freedom can produce a useful quantity of mechanical work.

In the case of a sailboat, the motion is a translatory one and the useful work is spent in overcoming the passive resistance of the water to the relative motion of the boat; in the case of a hydraulic machine (turbine) the motion is the rotation of a 'runner' and the work received on the 
rotation axis is spent (apart from a fraction absorbed by passive resistances, which is to be contained as effectively as possible) to generate mechanical power available for a variety of uses: milling and fulling in the most ancient applications, then forging and lifting of loads or fluids, and most recently for electric power generation.

In this last case, which will form the object of the present analysis, the freedom of motion of the solid runner is the rotation around an axis which is fixed with respect to the Earth surface. From this circumstance there derive some interesting features of the fluid-dynamic transformations taking place inside the runner of a reaction machine, of which it is possible to analyze the kinematic and dynamic broad lines by way of suitable schematizations and simplifications embodied in an elementary conceptual model. In what follows, it will be shown that this line of thought can provide provoking insights on a possible definition, for reaction turbines of low specific speed, of a basic runner geometry aiming at obtaining an orderly internal flow and a satisfactory 'best point' efficiency of the energy exchanges. Of course these insights are not sufficient to actually design a viable turbine runner; indeed, the definition of the shape of an industrially satisfactory reaction turbine calls not only for a more sophisticated flow-dynamics modeling, but also for a host of considerations of different nature (technological, economical...). Nonetheless, the present drastically simplified approach will bring to the fore some interesting peculiarities of the general aspects of the runner internal flow, as e.g. the often overlooked duality between two apparently mutually exclusive characters thereof (though actually two complementary faces of the same reality).

Let us now ask ourselves, "What approaches can be followed in trying to set up a mathematical model of the internal runner flow ?" The most comprehensive means would appear to be the integration of NAVIER-STOKES differential equations, in a moving system of reference and with the appropriate boundary conditions. This approach is fraught with pronounced difficulties of implementation, even not considering the problem (only partially tackled in some of the most advanced computational codes of Fluid Mechanics) of the modeling of turbulence. Besides, its application requires that the runner geometry be completely defined beforehand, and in this sense it does not lend itself to a direct design methodology, but rather requires a trial and error procedure, starting with a tentative geometry defined on the basis of experience and analogy and then gradually modifying it by evaluating, and if need be correcting, the alterations in outcome produced by each successive stage of refinement. Obviously this is a costly, time-consuming process, to be carried out by experienced personnel.

The present essay adopts the radically different assumption of a 'perfect fluid', in which the fluid is considered to be incompressible and inviscid, and its motion is assumed to be irrotational (with respect to a fixed reference frame) in the spaces between two adjacent vanes, the presence of vorticity being allowed only as indestructible filaments attached to the runner vanes and only free to slide along the vanes solid surfaces. In other words, the circulation around a closed circuit contained in an inter-vane space is taken to be zero, but it can be different from zero along a closed circuit encircling a vane. Moreover, the periodic disturbances caused by the passage of the (moving) runner vanes leading edges in front of the (fixed) distributor vanes trailing edges are altogether neglected (hypothesis of an infinite number of vanes). It will be seen that by selfevident alterations in the sign of some basic global parameters of the model it is possible to adopt the same type of schematization also for centrifugal pumps.

It will be shown that this approach gives the same global results as the elementary EULER theory of the reaction turbine; in addition it requires, to respect 'appropriate' boundary conditions, that the runner vanes be given a well-defined shape (which will be seen to depend only on the inlet and outlet angles). [The elementary, global EULER theory would instead be indifferent to the vane shape, i. e. it would give the same results for two runners having the same entry and exit angles, irrespective of the shape given to the vanes between runner entry and outlet, see Fig. 1a and Fig. 1b. In other words, the EULER theory implicitly assumes that the 
internal flow be 'perfectly guided' by the vane shape, however this shape might be contorted between inlet and outlet, provided that the inlet and outlet angles be kept fixed. Obviously this is not physically acceptable, implying e. g. that the two vane shapes represented in Fig. 1a and Fig. $1 \mathrm{~b}$ would give the same results. In this sense the present approach, though still a highly simplified one, is seen to represent a progress with respect to the EULER global model, inasmuch as by imposing the compatibility of the inter-vane flow with the model premises it is possible to define a theoretically 'acceptable' vane shape, to the exclusion of other ones].

The model described in the following is, indeed, internally consistent with such a simplified 'circulatory', perfect-fluid approach to the modeling of the internal flow in a reaction turbine or in a centrifugal pump. It will become clear that the approximations introduced in this model tend to be more acceptable for 'radial' machines. i.e. for Francis turbines of low specific speed. Though this kind of analysis cannot lead by itself to a realistic design procedure, it is endowed with an indisputable didactic and training value, enhancing a qualitative understanding of the physics of what happens inside the runner.

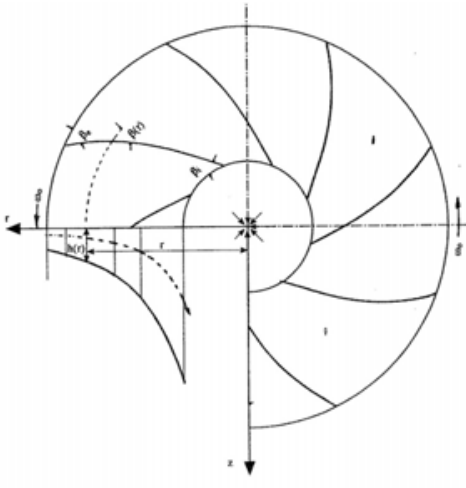

Fig. 1a

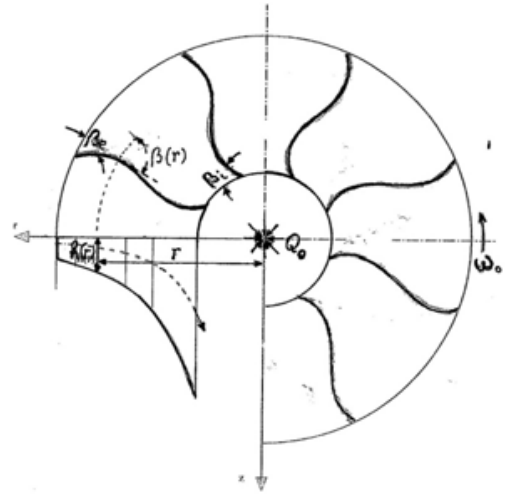

Fig. 1b

The angles $\beta_{e}$ and $\beta_{i}$ are the same in the two figures; the law of variation of the angle $\beta(r)$ with the radius $r$ is different in the two cases. According to the EULER global model the two runner shapes would be equally acceptable.

\section{THE SIMPLIFIED 3D, PERFECT-FLUID MATHEMATICAL MODEL OF THE IRROTATIONAL FLOW INSIDE THE RUNNER OF A REACTION TURBINE (FRANCIS TYPE) OF LOW SPECIFIC SPEED}

In this section the reader is invited to visualize a runner with an array of $N$ cylindrical vanes (the vane directrices being parallel to the rotation axis). The section of the vanes with planes normal to the rotation axis is defined by specifying the function $\beta=\beta(r)$, see Figs. 1a and $1 \mathrm{~b}$; the meridional runner section is defined by the function $h=h(r)$, see above-quoted figures. In subsequent algebraic developments, use is made of the product $h . s$ including a 'transparency' factor $s(r)<1$ expressing the ratio between the net passage section and the total cylindrical section. The runner is assumed to rotate with a uniform rotational speed $\omega_{0}$ and is filled with a centripetal flow of a perfect, incompressible fluid. The total discharge traversing the runner, $Q_{0}$, is expressed by:

(2.1) $Q_{0}=\pi \cdot R^{3} \cdot \omega_{0} \cdot \varphi_{0}$ where $\varphi_{0}$ is a dimensionless discharge index, $R$ is a reference radius (e. g. the radius $r_{e}$ of the runner inlet) and $\varphi_{0}$ has the character of a non-dimensional discharge index.

In order to simplify the developments, it is assumed that the axial depth of the inter-vane channels is given by $h(r) . s(r)=\frac{H_{e} \cdot r_{e}^{2}}{r^{2}}, s(r)$ being a 'transparency' index taking into account the finite thickness of the runner blades. It is $s(r)=1-t(r)$ with $t(r) \ll 1$. 
Moreover, to simplify things it is assumed that the runner is operating at its 'best efficiency' working point. Then with reference to Fig. 2 the absolute velocity at runner exit is radial and the velocity triangles should fulfill the following geometrical conditions:

at runner inlet section, entry without shock of the relative velocity with respect to the vane inlet angle $\beta_{e}$;

$$
c_{u_{e}}=\omega_{0} \cdot r_{e}-\frac{\pi \cdot R^{3} \cdot \omega_{0} \cdot \varphi_{0}}{2 \cdot \pi \cdot h_{e} \cdot r_{e} \cdot s_{e}} \operatorname{cotan} \beta_{e}, \text { or } c_{u_{e}}=\omega_{0} \cdot r_{e} \cdot\left(1-\frac{R^{3} \cdot \varphi_{0}}{2 \cdot h_{e} \cdot s_{e} \cdot r_{e}^{2}} \operatorname{cotan} \beta_{e}\right) ;
$$

- $\quad$ at runner exit section, outlet with radial absolute velocity, i. e.:

$$
\frac{R^{3} \cdot \varphi_{0}}{2 \cdot h_{i} \cdot s_{i} \cdot r_{i}^{2}} \cdot \operatorname{cotan} \beta_{i}=1
$$

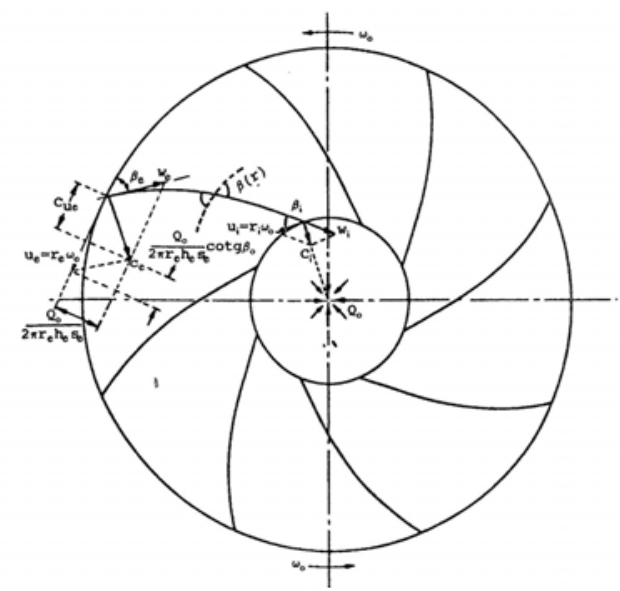

Fig. 2 - Inlet and exit velocity triangles of a runner vane during steady rotation.

Zenital view of the idealized runner and meaning of main symbols

The following steps aim to build up a model of the internal flow in which the absolute motion inside the inter-vane space is irrotational. This assumption implies:

a) that the vorticity of the relative flow (i.e. the flow as seen from a reference system rigidly rotating with the runner) be $-2 . \omega_{0}$, and

b) that any vorticity be tied to the solid surfaces of the vanes, i. e. that in each inter-vane space there be no free vortex filaments.

With reference to Fig. 2 the circulation $\Gamma_{v}$ around a closed circuit enclosing a vane is seen to be, assuming an identical situation for each vane and neglecting the radial clearance between distributor and runner:

$$
\Gamma_{v e}=\frac{2 . \pi \cdot r_{e} \cdot c_{u_{e}}}{N}=\frac{2 . \pi \omega_{0}}{N} \cdot\left(r_{e}^{2}-\frac{R^{3} \cdot \varphi_{0}}{2 \cdot h_{e} \cdot s_{e}} \operatorname{cotan} \beta_{e}\right)
$$

and likewise at radius $r, r_{i} \leq r \leq r_{e}$ :

$$
\Gamma_{v}(r)=\frac{2 . \pi \omega_{0}}{N} \cdot\left[r^{2}-\frac{R^{3} \cdot \varphi_{0}}{2 \cdot h(r) \cdot s(r)} \operatorname{cotan} \beta(r)\right]
$$

In a mathematical model of the assumedly 'generally irrotational' absolute runner flow the solid obstacles represented by each vane can be replaced, as is well known, by a system of sources, sinks and eddies -'singularities' completely determining the flow field- suitably distributed along the 'skeleton' profile of the vane. The local intensity of these singularities is determined by the condition that the fluid velocity be everywhere tangential with respect to the vane surface. In accord with that, let us assume that each one of our $N$ vanes is replaced by a distribution of eddies totaling the circulation $\Gamma_{v}$ of Eq. (2.4) for each vane, while a diffused sink of discharge $-Q_{0}$ absorbs the centripetal flow in the center. Then let us apply another well-known theorem of Perfect Fluid Dynamics (see e. g. Horace LAMB's “Hydrodynamics”), stating that a vortex filament immersed in the motion field of a perfect fluid moves with a local (absolute) velocity 
which is determined by all the singularities of the motion field. In the proposed model, it is assumed, in accord with the quoted theorem, that these singularities stay on the vanes 'as if' they were free and therefore were carried along in the general motion field.

The combined fulfilling of these two constraints requires that:

c) in the general 'advection' motion field generated by the centripetal discharge $Q_{0}$ and by the presence of the $N$ vane-attached eddies, each single vane be the bearer of a macroscopic eddy of circulation $\Gamma_{v}$. These eddies, regarded as 'freely following' the general motion, remain all the time tied to the skeleton profile of the respective vanes; any other hypothesis would, in fact, be tantamount to accepting a separation of the flow from the vanes, contrary to the assumption of 'best efficiency' regime of the runner.

d) the radial distribution of vorticity along each skeleton vane profile, given by:

$$
\gamma(r)=\frac{d \Gamma_{v}(r)}{d r}, \text { where } \int_{r_{i}}^{r_{e}} \gamma(r) \cdot d r=\Gamma_{v e}, \int_{r_{i}}^{r} \gamma(r) \cdot d r=\Gamma_{v}(r)
$$

remain unchanged during the motion.

These conditions of our mathematical model will be henceforth denoted as the 'self-replicating conditions' of our idealized runner flow. It remains to express them in a form viable for obtaining further insights, in particular for the determination, if possible, of the plan shape of the vane radial 'skeleton' profile. To this end, it is necessary first of all to proceed from the foregoing developments towards the expression of the general advection motion field of point c) here above.

With reference to Fig. 3 assume that the meridional section of the runner inner flow is contained between the two solid surfaces:

$$
z=0 \text { (runner cover) and } z(r)=\frac{H_{e}}{r^{2}} \cdot r_{e}^{2} \text { (runner 'mantle' or 'belt'). }
$$

The inter-vane flow field can be approximated by the superposition of two 'generally irrotational' components:

a first, cylindrically symmetric flow field whose potential is expressed by:

$$
\Phi_{1}=\frac{A}{4} \cdot \omega_{0} \cdot \varphi_{0} \cdot\left(z^{2}-\frac{r^{2}}{2}\right)=\frac{R^{3}}{2 \cdot r_{e}^{2} \cdot H_{e}} \cdot \omega_{0} \cdot \varphi_{0} \cdot\left(z^{2}-\frac{r^{2}}{2}\right), \quad \text { where } A=\frac{2 \cdot R^{3}}{r_{e}^{2} \cdot h_{e} \cdot s_{e}}=\frac{2 \cdot R^{3}}{r_{e}^{2} \cdot H_{e}},
$$

giving the two components of velocity:

$$
V_{1 r}=\frac{\partial \Phi_{1}}{\partial r}=-\frac{R^{3}}{2 \cdot r_{e}^{2} \cdot H_{e}} \cdot \omega_{0} \cdot \varphi_{0} \cdot r
$$

(radial component of the first flow field) and

$$
V_{1 z}=\frac{\partial \Phi_{1}}{\partial z}=\frac{R^{3}}{r_{e}^{2} \cdot H_{e}} \cdot \omega_{0} \cdot \varphi_{0} \cdot z
$$

(axial component of the first flow field); see Fig. 3.

- a second, vortex-dominated (but still irrotational in the inter-vane spaces) flow field produced by a distribution of indefinite, rectilinear vortex filaments parallel to the runner axis and localized on the vane skeleton profiles, the distribution of vorticity along each of the $N$ vanes being expressed, as per Eq.(2.5), as:

$$
\gamma(r)=\frac{d \Gamma_{v}(r)}{d r}
$$

this radial density of vorticity will be defined further on so as to comply with the self- replicating conditions, while its velocity components are evaluated as if at each radius $r$ the $N$ elementary 
vortices of circulation $\gamma(r) . d r$ were uniformly distributed over the circumference of radius $r$, this last assumption being acceptable if $N$ be sufficiently large (say of the order of 20).

Therefore the approximated velocity components of the second field are evaluated as:

$$
\begin{array}{ll}
V_{2 c}(r)=\frac{N}{2 \cdot \pi \cdot r} \cdot \int_{r_{i}}^{r} \gamma(r) \cdot d r & \text { for } r_{i} \leq r \leq r_{e} \text {, circumferential component; } \\
V_{2 r} \cong 0, V_{2 z} \cong 0 & \text { respectively radial and axial components. }
\end{array}
$$

Let us point out that the radial velocity (2.8) of the first flow field is independent from $z$, and that the radial velocity (2.12) of the second flow field is negligible if the number $N$ of vanes is sufficiently high (see above), while its circumferential velocity (2.11) has been assumed to be approximately independent from $z$, i. e. to be a function only of $r$. In this way an indefinitely extended total flow field is approximately described, by 'cutting out' a finite portion of it contained between cover and belt of the runner, i. e. between the surfaces:

$$
z=0, z=\frac{2 \cdot R^{3}}{A \cdot r^{2}}=\frac{H_{e} \cdot r_{e .}^{2}}{r^{2}}, r_{i} \leq r \leq r_{e} \text {, (since } A=\frac{2 \cdot R^{3}}{r_{e}^{2} \cdot h_{e} \cdot s_{e}}=\frac{2 \cdot R^{3}}{r_{e}^{2} \cdot H_{e}} \text { ) }
$$

The resulting motion field is adequate to represent, albeit in an admittedly approximated way, the inter-vane motion field inside a Francis-type runner of low specific speed with $N$ vanes.

Now two conditions remain to be expressed:

- that the vortex total and local circulation is preserved during the general field motion ('Vortex Conservation condition', in short VCC),

- that the distribution of vorticity along each vane skeleton profile is left unchanged during the general 'advection motion' ('Self-Replicating Condition', in short SRC).

From the two conditions, recalling Eqs. (2.1) and (2.4.I), i. e. $Q_{0}=\pi \cdot R^{3} \cdot \omega_{0} \cdot \varphi_{0}, N \cdot \Gamma_{v e}=$ 2. $\pi \cdot r_{e} \cdot c_{u_{e}}=2 \cdot \pi \omega_{0} \cdot\left(r_{e}^{2}-\frac{R^{3} \cdot \varphi_{0}}{2 \cdot h_{e} \cdot s_{e}} \operatorname{cotan} \beta_{e}\right)$, it is straightforward to obtain the following two equations:

$$
\begin{aligned}
& \frac{N}{2 . \pi \cdot r} \cdot \int_{r_{i}}^{r} \gamma(r) \cdot d r=\omega_{0} \cdot r-\frac{Q_{0} \cdot \operatorname{cotan} \beta(r)}{2 . \pi \cdot r \cdot h(r) \cdot s(r)}, \quad \text { or } \quad\left[\begin{array}{ll}
\text { see } & (2.1)
\end{array}\right] \quad \frac{N}{2 . \pi \cdot \omega_{0} \cdot r} \int_{r_{i}}^{r} \gamma(r) \cdot d r+ \\
& \text { R3.cotan } \beta \text { r2.r.hr.sr. } \varphi 0=r \\
& \frac{d\left[\gamma(r) \cdot\left(V_{1 r}+V_{2 r}\right)\right]}{d r}=0 \text {, or [see (2.8) and (2.12)] }-\frac{A}{4} \cdot \omega_{0} \cdot \varphi_{0} \cdot\left[r \cdot \frac{d[\gamma(r)]}{d r}+\gamma(r)\right]=0 \text {, }
\end{aligned}
$$

Summing up: from $r \cdot \frac{d[\gamma(r)]}{d r}+\gamma(r)=0$, i. e. $\frac{d}{d r} \ln [\gamma(r)]=\frac{1}{\gamma(r)} \cdot \frac{d[\gamma(r)]}{d r}=-\frac{1}{r}=-\frac{d}{r}[\ln (r)]$, so that:

$$
\begin{aligned}
& \Gamma_{v e}=\frac{2 \cdot \pi \cdot r_{e} \cdot c_{u_{e}}}{N}=\frac{2 \cdot \pi \omega_{0}}{N} \cdot\left(r_{e}^{2}-\frac{R^{3} \cdot \varphi_{0}}{2 \cdot h_{e} \cdot s_{e}} \operatorname{cotan} \beta_{e}\right)=\int_{r_{i}}^{r_{e}} \gamma(r) \cdot d r=r_{i} \cdot \gamma\left(r_{i}\right) \cdot \ln \frac{r_{e}}{r_{i}} \\
& \Gamma_{v}(r)=\frac{2 \cdot \pi \cdot r \cdot c_{u}(r)}{N}=\frac{2 \cdot \pi \omega_{0}}{N} \cdot\left[r^{2}-\frac{R^{3} \cdot \varphi_{0}}{2 \cdot h(r) \cdot s(r)} \operatorname{cotan} \beta(r)\right]=r_{i} \cdot \gamma\left(r_{i}\right) \cdot \ln \frac{r}{r_{i}}
\end{aligned}
$$

Fig. 3 - Meridional section of the runner; radial and axial components of velocity 
Indeed, from Eq. (2.15) it comes successively:

$$
\begin{aligned}
& r \cdot \frac{d[\gamma(r)]}{d r}+\gamma(r)=0 \\
& \frac{\frac{d[\gamma(r)]}{d r}}{\gamma(r)}=-\frac{1}{r} \quad \text { and } \ln \gamma(r)=-\ln r \\
& \gamma(r)=\gamma\left(r_{i}\right) \cdot \frac{r_{i}}{r}
\end{aligned}
$$

Then from Eq. (2.14) $\frac{N}{2 \cdot \pi \cdot \omega_{0} \cdot r} \int_{r_{i}}^{r} \gamma(r) \cdot d r+\frac{R^{3} \cdot \operatorname{cotan} \beta(r)}{2 \cdot r \cdot h(r) \cdot s(r)} \cdot \varphi_{0}=r$; assuming [see Eq. (2.6)]: $h(r) . s(r)=\frac{H_{e}}{r^{2}} \cdot r_{e}^{2}$

and for the shape of the skeleton vane profile:

from which:

$$
\operatorname{cotan} \beta(r)=\frac{2 \cdot H_{e} \cdot r_{e}^{2}}{R^{3} \cdot \varphi_{0}} \cdot\left(1-\frac{N \cdot \gamma\left(r_{i}\right) \cdot r_{i}}{2 \cdot \pi \cdot \omega_{0} \cdot r^{2}} \cdot \ln \frac{r}{r_{i}}\right),
$$

$$
\begin{aligned}
& \operatorname{cotan} \beta\left(r_{I}\right)=\frac{2 \cdot H_{e} \cdot r_{e}^{2}}{R^{3} \cdot \varphi_{0}}, \\
& \operatorname{cotan} \beta\left(r_{e}\right)=\frac{2 \cdot H_{e} \cdot r_{e}^{2}}{R^{3} \cdot \varphi_{0}} \cdot\left(1-\frac{N \cdot \gamma\left(r_{i}\right) \cdot r_{i}}{2 \cdot \pi \cdot \omega_{0} \cdot r_{e}^{2}} \cdot \ln \frac{r_{e}}{r_{i}}\right) \\
& \operatorname{cotan} \beta\left(r_{e}\right)=\operatorname{cotan} \beta\left(r_{I}\right) \cdot\left(1-\frac{N \cdot \gamma\left(r_{i}\right) \cdot r_{i}}{2 \cdot \pi \cdot \omega_{0} \cdot r_{e}^{2}} \cdot \ln \frac{r_{e}}{r_{i}}\right)
\end{aligned}
$$

NOTE: It is convenient to express the profile in polar coordinates $r, \vartheta$ :

$$
\begin{aligned}
& \operatorname{cotan} \beta(r)=\frac{r \cdot d \vartheta}{d r}=\frac{2 \cdot H_{e} \cdot r_{e}^{2}}{R^{3} \cdot \varphi_{0}} \cdot\left[1-\frac{N \cdot \gamma\left(r_{i}\right) \cdot r_{i}}{2 \cdot \pi \cdot \omega_{0} \cdot r^{2}} \cdot \ln \frac{r}{r_{i}}\right] \\
& \frac{d \vartheta}{d r}=\frac{2 \cdot H_{e} \cdot r_{e}^{2}}{R^{3} \cdot \varphi_{0}} \cdot\left[\frac{1}{r}-\frac{N \cdot \gamma\left(r_{i}\right) \cdot r_{i}}{2 \cdot \pi \cdot \omega_{0} \cdot r^{3}} \cdot \ln \frac{r}{r_{i}}\right] \\
& \vartheta(r)=\vartheta\left(r_{i}\right)+\frac{2 \cdot H_{e} \cdot r_{e}^{2}}{R^{3} \cdot \varphi_{0}} \cdot\left[\ln \frac{r}{r_{i}}+\frac{N \cdot \gamma\left(r_{i}\right) \cdot r_{i}}{4 \cdot \pi \cdot \omega_{0} \cdot r^{2}}\left(\ln \frac{r}{r_{i}}+\frac{1}{2}-\frac{r^{2}}{2 \cdot r_{i}^{2}}\right)\right]
\end{aligned}
$$

Moreover, from Eq. (2.16) it comes for the distribution of vorticity density $\gamma(r)$ as a function of the local radius:

$$
\gamma\left(r_{i}\right)=\frac{2 \cdot \pi \cdot \omega_{0} \cdot r_{e}^{2}}{N \cdot r_{i} \cdot \ln \frac{r_{e}}{r_{i}}}\left[1-\frac{\operatorname{cotan} \beta\left(r_{e}\right)}{\operatorname{cotan} \beta\left(r_{I}\right)}\right]=\frac{\Gamma_{v}\left(r_{e}\right)}{r_{i} \cdot \ln \frac{r_{e}}{r_{i}}}
$$

For $\beta\left(r_{e}\right)>\beta\left(r_{i}\right) \gamma(r)>0$ (turbine) and for $\beta\left(r_{e}\right)<\beta\left(r_{i}\right) \gamma(r)<0$ (pump).

\section{THE MECHANICAL PERFORMANCE OF THE IDEAL TURBOMACHINE ACCORDING TO THE CIRCULATORY MODEL}

It is now possible to express the moment of the lift forces exerted by the radial ${ }^{1}$ runner flow over the vane-bound vortices, thanks to the LAGALLY formula for the theoretical lift. Taking $s(r) \cong 1$ :

$$
F_{v}=-\rho \cdot \int_{r_{i}}^{r_{e}} h(r) \cdot \frac{-Q_{0}}{2 \cdot \pi \cdot r \cdot h(r) \cdot s(r)} \cdot \gamma(r) \cdot d r=\frac{\rho \cdot Q_{0}}{2 \cdot \pi} \cdot \int_{r_{i}}^{r_{e}} \frac{\gamma(r)}{r} \cdot d r=\frac{\rho \cdot Q_{0}}{2 \cdot \pi} \cdot \gamma\left(r_{i}\right) \cdot r_{i} \cdot \int_{r_{i}}^{r_{e}} \cdot \frac{d r}{r^{2}}
$$

\footnotetext{
${ }^{1}$ The circumferential component of the runner flow exerts on the vanes radial LAGALLY forces that do not contribute to the torque $M$.
} 


$$
F_{v}=\frac{\rho \cdot Q_{0}}{2 \cdot \pi} \cdot \gamma\left(r_{i}\right) \cdot r_{i} \cdot\left(\frac{1}{r_{i}}-\frac{1}{r_{e}}\right)=\rho \cdot R^{3} \cdot \omega_{0}^{2} \cdot \varphi_{0} \cdot \frac{r_{e}^{2}}{N \cdot r_{i} \cdot \ln \frac{r_{e}}{r_{i}}}\left[\frac{1-\operatorname{cotan} \beta\left(r_{e}\right)}{\operatorname{cotan} \beta\left(r_{I}\right)}\right]
$$

and the moment exerted by the flow over the $N$ vanes, i. e. on the runner solid structure:

$$
\begin{aligned}
& M=N \cdot \rho \cdot \int_{r_{i}}^{r_{e}} h(r) \cdot \frac{Q_{0}}{2 \cdot \pi \cdot r \cdot h(r)} \cdot \gamma(r) \cdot r \cdot d r=N \cdot \frac{\rho \cdot Q_{0}}{2 \cdot \pi} \cdot \gamma\left(r_{i}\right) \cdot r_{i} \cdot \int_{r_{i}}^{r_{e}} \cdot \frac{d r}{r}= \\
& N \cdot \frac{\rho \cdot Q_{0}}{2 \cdot \pi} \cdot \gamma\left(r_{i}\right) \cdot r_{i} \cdot \ln \frac{r_{e}}{r_{i}} \\
& M=\rho \cdot R^{3} \cdot \omega_{0}^{2} \cdot \varphi_{0} \cdot \pi \cdot r_{e}^{2} \cdot\left[1-\frac{\operatorname{cotan} \beta\left(r_{e}\right)}{\operatorname{cotan} \beta\left(r_{I}\right)}\right]
\end{aligned}
$$

The theoretical power that is collected on the rotation axis of the machine is therefore:

$$
W=M \cdot \omega_{0}=\rho \cdot R^{3} \cdot \omega_{0}^{3} \cdot \varphi_{0} \cdot \pi \cdot r_{e}^{2} \cdot\left[1-\frac{\operatorname{cotan} \beta\left(r_{e}\right)}{\operatorname{cotan} \beta\left(r_{I}\right)}\right]=\rho \cdot g \cdot H_{0} \cdot \pi \cdot R^{3} \cdot \omega_{0} \cdot \varphi_{0}
$$

from which the theoretical head $H_{0}$ :

$$
H_{0}=\frac{\omega_{0}^{2} \cdot r_{e}^{2}}{g} \cdot\left[1-\frac{\operatorname{cotan} \beta\left(r_{e}\right)}{\operatorname{cotan} \beta\left(r_{I}\right)}\right]
$$

It is noteworthy that Eqs. (3.1) to (3.4) are the same as can be derived from the EULER global approach. The additional information that can be obtained from the present theory, based on the circulatory approach, shows in a clear way that the shape of the vane profile between runner entry and exit is not indifferent. Indeed, for an arbitrary vane profile respecting the given entry and exit angles the self-replicating conditions for the vane-attached vorticity distribution will not be respected and flow separation from the vane surfaces will have to be expected.

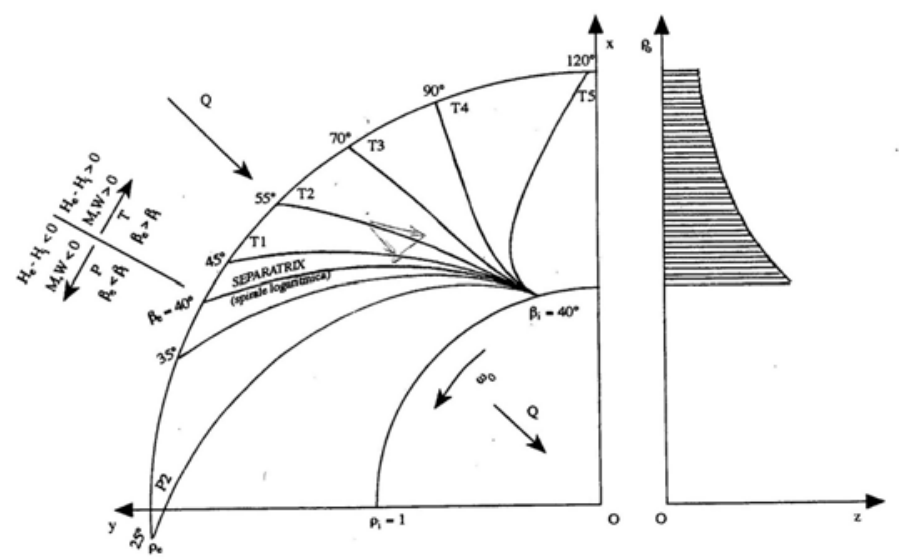

Fig. 4 - Several skeleton vane profiles for a single exit angle, $\beta\left(r_{i}\right)=40^{\circ}$, and different inlet angles $\beta\left(r_{e}\right)>\beta\left(r_{e}\right)$ [From Eq. (2. 24)].

The profile marked 'SEPARATRIX' is a logarithmic spiral $\left[\beta\left(r_{e}\right)=\beta(r)=\beta\left(r_{i}\right)\right]$ and corresponds to a hypothetical machine running at zero power (runaway mode, axial inlet and exit velocities). The profiles with $\beta(r)>\beta\left(r_{i}\right)$ are typical of turbine runners, those with $\beta(r)<\beta\left(r_{i}\right)$ are typical of pump runners (in this example, 'centripetal'-type pumps not used in practice).

\section{CONCLUSIONS}

The above simplified mathematical model of the runner of a low specific speed reaction hydraulic turbo machine shows some of the advantages of the 'circulatory' approach for a better comprehension of some of the essential features of the internal fluid flow. The paradigm of the perfect fluid, of the irrotational flow in the inter-vane spaces, of the vane-fluid kinematics and dynamics through the replacement of each vane with a self-replicating system of vorticity distributions attached to the vane allows to gain some interesting insights (albeit schematic ones of the energy transformations taking place inside a Francis-type runner. 
It is possible to extend the present theory to the analysis of the internal flow at off-design regimes of the machine (exit velocity no longer radial) (see original paper in Italian).

Further developments of potential interest appear as feasible. In particular, modeling the presence of a 'vortex rope' downstream of the runner exit section could be attempted. Also, the modeling of some instationary types of regime, such as rotating stalls or inter-vane channel block, vane-detached flow etc., could conceivably profit from their representation introducing in the model the presence of concentrated or distributed vortices, co-rotating or moving relative to the runner. The present essay is viewed as a simple introduction to a variety of more esoteric schematizations of engineering interest.

First published (in Italian) May 1993; English version December 2016

\section{SHORT REFERENCE LIST}

[1] Lamb, H. (1996). Hydrodynamics. Cambridge.

[2] Boussuges, S. (1955). Machines Hydrauliques, ENSHERG, Grenoble.

[3] DE ST. MARC, S. (1955). Hydrodynamique Graphique, ENSHERG, Grenoble.

[4] Fanelli, M. (1990). Vortex-dominated Flow in Centripetal Runners and Draft-Tubes, Symposium IAHR, Belgrade.

[5] Fanelli, M. (1971). Remarks on the circulatory theory of turbomachinery, Internal Report of CRIS /ENEL, (in Italian)

[6] Luu, T. S. \& Viney, B. (1989). Schème tourbillonnaire unifié des problèmes direct et inverse de l'écoulement subcavitant dans une turbomachine, Symposium SHF, Lyon. 